\title{
Thank you to the reviewers
}

The guest editors would like to thank those listed below for constructive and insightful reviews of the manuscripts. Your expertise has contributed importantly to this special issue.

Anne Lise Brantsæter, Norwegian Institute of Public Health, Oslo, Norway

Camilla Lindvall Dahlgren, Oslo University Hospital, Oslo, Norway

Berit Granum, Norwegian Institute of Public Health, Oslo, Norway

Marte Handal, Norwegian Institute of Public Health, Oslo, Norway

Kristin Holvik, Norwegian Institute of Public Health, Oslo, Norway

Ann-Charlotte Iversen, Norwegian University of Science and Technology (NTNU) Trondheim, Norway

Kim Stene-Larsen, Norwegian Institute of Public Health, Oslo, Norway

Rolv Terje Lie, University of Bergen, Bergen, Norway

Helle Margrete Meltzer, Norwegian Institute of Public Health, Oslo, Norway

Wenche Nystad, Norwegian Institute of Public Health, Oslo, Norway

Randi Selmer, Norwegian Institute of Public Health, Oslo, Norway

Olav Spigset, St. Olavs Hospital, Trondheim, Norway

Nina Stenberg, Norwegian Institute of Public Health, Oslo, Norway

Lars Christian Stene, Norwegian Institute of Public Health, Oslo, Norway

Katrine Strandberg Larsen, University of Copenhagen, Copenhagen, Denmark

Pål Surén, Norwegian Institute of Public Health, Oslo, Norway

Anne Johanne Søgaard, Norwegian Institute of Public Health, Oslo, Norway

Eivind Ystrøm, Norwegian Institute of Public Health, Oslo, Norway

Ragnhild Elise Ørstavik, Norwegian Institute of Public Health, Oslo, Norway

Anne-Siri Øyen, Norwegian Institute of Public Health, Oslo, Norway 